\title{
Sedimentación de partículas con distribución de tamaño fractal
}

\author{
Roberto R. Filgueira ${ }^{1}$, Yakov A. Pachepsky' ${ }^{2}$, Lidia L. Fournier'1, Mirta G. García ${ }^{3}$ \& Guillermo O. Sarli'
}

\begin{abstract}
RESUMEN
Desde hace varios años, el modelo de fragmentación fractal ha atraido la atención de los investigadores, como un camino lógico para describir e interpretar distribuciones de partículas observadas. El análisis textural de un suelo ha mostrado ser muy importante, pues se utiliza para diagnosticar y predecir el funcionamiento y uso del mismo. Los métodos más populares para determinar la textura han sido los de sedimentación en agua utilizando el hidrómetro o la pipeta. Ambos tienen como objetivo encontrar la fracción de masa de partículas que se encuentran en suspensión a tiempos prefijados y relacionarla con los diámetros de las mismas. En este trabajo se ha desarrollado una nueva función potencial que relaciona la fracción de masa en suspensión con el tiempo de sedimentación. Utilizando la misma se puede determinar la dimensión fractal de fragmentación de una distribución de partículas en sedimentación. La nueva ecuación ha sido chequeada con datos propios obtenidos por el hidrómetro de Bouyoucos y otros publicados en la literatura, obtenidos mediante la pipeta de Robinson. El acuerdo logrado entre la teoría y los datos experimentales, mediante la técnica de regresión no lineal, ha sido excelente. Los valores de la dimensión fractal de fragmentación resultaron entre 2,404 y 2,512, para muestras de La Plata, Argentina, y entre 2,434 y 2,819 para los suelos de California, USA. El coeficiente de determinación, $R^{2}$, fue en todos los casos mayor que 0,9 .
\end{abstract}

Palabras-clave: suelos, textura, fragmentación

\section{Sedimentation of fractal size distribution particles}

\begin{abstract}
Since several years the fractal fragmentation model has attracted the attention of researchers, as a logic way to describe and interprete observed particle size distributions. Textural analysis has shown to be very important because of its usefulness in the dignosis and inferences about soil functioning and use. Most popular methods of textural analysis employ sedimentation of particles in water using the hydrometer or the pipet. Both have the objective of determining the particle fraction remaining in suspension at predetermined time and to relate them with particle diameters. In the present work a new power law relationship between the mass fraction in suspension and the time was developed. Using this relationship it was possible to determine the fragmentation fractal dimension of a set of particles in sedimentation. The new equation has been checked with data obtained in this research by the Bouyoucos's hydrometer, and others published in the literature, using the Robinson's pipet method. The agreement between the model and the experimental data, using non linear regression, was excellent. Resulting fractal fragmentation dimensions ranged from 2.404 to 2.512, for samples from La Plata, Argentina, and between 2.434 and 2.819 for soils from California, USA. Determination coefficients, $\mathrm{R}^{2}$, were always higher than 0.9 .
\end{abstract}

Key words: soils, texture, fragmentation

${ }^{1}$ Facultad de Ciencias Agrarias y Forestales, UNLP, Calles 60 y 119, CC 31, 1900 La Plata, Argentina. Fone: +54 221 4236758. Email: filgueira@infovia.com.ar; Ilfournier@infovia.com.ar; gsarli@ceres.agro.unlp.edu.ar

2 US Dept. of Agriculture, Animal Waste Pathogen Laboratory, Bldg. 173, Rm. 203, BARC-EAST, Powder Mill Road, Beltsville, MD 20705. USA. Fone: +1 301 504 7468. Email: ypachepsky@anri.barc.usda.gov

${ }^{3}$ Facultad de Ciencias Agrarias y Forestales, UNLP, Calles 60 y 119, CC 31, 1900 La Plata, Argentina. Fone: + 54221 4236758. Email: mycsuelos@ceres.agro.unlp.edu.ar 


\section{INTRODUCCION}

Los suelos se han formado a partir de material primario fragmentado por meteorización, dando origen a una distribución de tamaño de partículas y agregados, que frecuentemente es interpretada como una función acumulativa, ya sea del número de partículas mayores que un cierto diámetro, o como la masa menor que un cierto diámetro. Una de las relaciones matemáticas más conocidas para este proceso es la siguiente (Mandelbrot, 1982; Turcotte, 1986):

$$
\mathrm{N}(\mathrm{r}>\mathrm{R})=\mathrm{k} \mathrm{R}^{-\mathrm{D}}
$$

donde N ( $r>R$ ) es el número de objetos por unidad de volumen que tienen un radio mayor que un valor cualquiera $\mathrm{R}$, mientras que $\mathrm{k}$ y $\mathrm{D}$ son constantes. Esta relación potencial define lo que se denomina un fractal probabilístico, también llamado fractal de fragmentación, siendo D la dimensión fractal de fragmentación (Baveye \& Boast, 1998). En este caso, es esperable que el modelo sea válido dentro de un rango limitado de radios, dado que no es un fractal matemático. Turcotte (1986) propuso un modelo para el proceso de fragmentación de un cubo, que dividido hipotéticamente en ocho partes iguales puede fragmentarse en ocho cubos más pequeños. Cada cubo resultante puede a su vez dividirse en otros ocho, y así sucesivamente. La fragmentación del cubo tiene una probabilidad $\mathrm{p}$ de ocurrir, y se considera constante para todos los órdenes de tamaño. La probabilidad máxima sería $\mathrm{p}=1$, que implica una división en ocho cubos más chicos, y la mínima sería $\mathrm{p}=1 / 8$, para el desprendimiento de un solo cubo de los ocho posibles. En estas condiciones se dedujo la siguiente relación.

$$
\mathrm{D}=\frac{\log (8 \mathrm{p})}{\log 2}
$$

donde $\mathrm{D}$ es la dimensión fractal, que puede variar entre $0 \mathrm{y}$ 3 , y p la probabilidad de fragmentación.

Para partículas del suelo, Tyler \& Wheatcraft (1992) sugirieron otra forma de expresar la Ec. 1, introduciendo la fracción de masa en vez del número de partículas. La ventaja consiste en que, experimentalmente, es más sencillo y preciso obtener fracciones de masa que número de partículas. Con este modelo, la masa acumulada de las partículas con un radio mayor que un valor $\mathrm{R}$ es:

$$
\mathrm{M}(\mathrm{r}>\mathrm{R})=\rho_{\mathrm{p}} \mathrm{C}_{\mathrm{m}}\left[1-\left(\frac{\mathrm{R}}{\lambda_{\mathrm{m}}}\right)^{3-D}\right]
$$

donde $\mathrm{C}_{\mathrm{m}}$ y $\lambda_{\mathrm{m}}$ son constantes.

La masa total de la distribución de estas partículas es:

$$
\mathrm{M}_{\mathrm{T}}=\mathrm{M}(\mathrm{r}>0)=\rho_{\mathrm{p}} \mathrm{C}_{\mathrm{m}}\left[1-\left(\frac{0}{\lambda_{\mathrm{m}}}\right)^{3-\mathrm{D}}\right]=\rho_{\mathrm{p}} \mathrm{C}_{\mathrm{m}}
$$

La Ec. 3 puede ser normalizada, dividiendo por la Ec. 4, con lo que se obtiene la fracción de masa de partículas mayores que un valor $\mathrm{R}$ :

$$
\frac{\mathrm{M}(\mathrm{r}>\mathrm{R})}{\mathrm{M}_{\mathrm{T}}}=\left[1-\left(\frac{\mathrm{R}}{\lambda_{\mathrm{m}}}\right)^{3-\mathrm{D}}\right]
$$

Para evaluar la constante $\lambda_{\mathrm{m}}$ suponemos un límite superior para el comportamiento fractal, $\mathrm{r}=\mathrm{R}_{\mathrm{Lsup}}$. Para el caso de partículas del suelo, este límite sería el de la arena muy gruesa. Cuando $r=R_{L s u p}$ se cumplirá que $M\left(r>R_{L s u p}\right) / M_{T}=0$, es decir, ya no habrá partículas por encima de este límite. De la Ec. 5 resulta entonces $R_{\text {Lsup }}=\lambda_{\mathrm{m}}$.

En el caso del experimento de sedimentación es común obtener la fracción de masa de partículas que aún permanecen en la suspensión, es decir, $\mathrm{M}\left(\mathrm{r}<\mathrm{R}_{\mathrm{Lsup}}\right) / \mathrm{M}_{\mathrm{T}}$. Podemos transformar fácilmente la Ec. 5, de la siguiente forma:

$$
\frac{M(r<R)}{M_{T}}=1-\frac{M(r>R)}{M_{T}}=\left(\frac{R}{R_{L s u p}}\right)^{3-D}
$$

Este modelo ha sido aplicado también a muestras de suelos por otros autores (Bitelli et al., 1999; Filgueira et al., 2002a,b). Bitelli et al. (1999) estudiaron la distribución de tamaño de partículas de varios suelos mediante la técnica de tamizado en húmedo, la pipeta, y la difracción de la luz, en el rango 0,1 - $2000 \mu \mathrm{m}$. Estos autores identificaron en las distribuciones de tamaño de partículas, tres rangos separados por diferentes dimensiones fractales, que llamaron dominios arcilla, limo y arena, respectivamente. Los resultados mostraron que las dimensiones fractales obtenidas eran muy diferentes entre los dominios y aún dentro de cada uno de éstos. Filgueira et al. (2002b) investigaron el comportamiento de la distribución de tamaño de partículas de un suelo, a profundidades de 0 a $400 \mathrm{~mm}$, mediante la técnica de Bouyoucos y con la aproximación fractal basada en la fracción de masa acumulada respecto del diámetro de las partículas (Ec. 6). Se corroboró que la distribución de masa de partículas acumulada versus diámetro sigue una ley potencial, en todos los casos. La dimensión fractal de fragmentación del dominio limo creció a medida que aumentó la profundidad del suelo.

Cuando un cuerpo cualquiera se mueve dentro de un fluido viscoso, el medio ejerce una fuerza de oposición al avance. De igual manera, si mantenemos el cuerpo quieto y es el fluido el que se mueve alrededor del objeto, éste siente una fuerza debida a esta corriente.

Stokes (1851) investigó el caso particular del movimiento de una esfera en un medio viscoso y encontró una expresión para la fuerza viscosa que se opone al movimiento, para el caso en que este movimiento (esfera respecto al fluido) sea tal que se conserven las condiciones de flujo laminar.

La expresión matemática de esta fuerza es:

$$
\mathrm{F}_{\mathrm{v}}=6 . \pi \cdot \eta \cdot \mathrm{R} \cdot \mathrm{v}
$$

donde $\eta$ es el coeficiente absoluto de viscosidad del líquido, 
$\mathrm{R}$ es el radio de la esfera, y $v$ la velocidad de la esfera respecto al fluido. La Ec. 7 es conocida como Ley de Stokes. El estudio de la caida de un cuerpo esférico en el interior de un líquido viscoso, en reposo, nos muestra que éste alcanza una velocidad de caída constante llamada velocidad límite. Planteando el sistema de fuerzas que actúan sobre el cuerpo en la caída, se deduce la siguiente expresión:

$$
\mathrm{V}_{\lim }=\frac{2}{9} \frac{\mathrm{R}^{2} \mathrm{~g}}{\eta}\left(\rho_{\mathrm{c}}-\rho_{\mathrm{L}}\right)
$$

donde $\mathrm{v}_{\text {lim }}$ es la velocidad límite, $\mathrm{R}$ el radio de la esfera, $g$ la aceleración de la gravedad, $\eta$ el coeficiente de viscosidad absoluta del líquido, $\rho_{\mathrm{c}}$ y $\rho_{\mathrm{L}}$ son las densidades de la esfera y del líquido, respectivamente. La velocidad límite se puede obtener fácilmente, midiendo una cierta distancia de caída $\mathrm{h}$, tomando el tiempo empleado t. Luego, reemplazando $\mathrm{v}_{\text {lim }}$ por $\mathrm{h} / \mathrm{t}$ en la Ec. (8) y reagrupando nos queda:

$$
R=\left(\frac{9 \eta h}{2 g\left(\rho_{c}-\rho_{L}\right) t}\right)^{\frac{1}{2}}
$$

Una aplicación muy difundida de la Ec. 9 es su uso en sedimentación, para separar partículas de diferentes radios, que están moviéndose en un líquido. Un ejemplo es el caso del estudio de la granulometría de un suelo; siendo los métodos más conocidos y aceptados los del hidrómetro de Bouyoucos y el de la pipeta de Robinson (Gee \& Bauder, 1986). En estos casos se supone que las partículas del suelo son esferas con un radio equivalente al resultante de la Ec. 9. Kaddah (1974) realizó una investigación comparativa detallada de 24 suelos de California, utilizando las técnicas del hidrómetro y la pipeta, llegando a la conclusión de que tomando los recaudos necesarios ambos son equivalentes. Este autor publicó sus resultados en forma de tabla donde se daban los porcentajes de partículas que permanecían en suspensión acuosa, para diámetros de las mismas menores que $20,10,5,2$, y $1 \mu \mathrm{m}$. También desarrolló una metodología gráfica para convertir los datos del experimento de sedimentación a distintas temperaturas, en diámetros de partículas y porcentajes, y viceversa.

No hay antecedentes de que el modelo de fragmentación haya sido relacionado con la distribución de masa de partículas en función del tiempo de sedimentación en un líquido.

El objetivo de este trabajo fue encontrar una ecuación que relacione la distribución fractal de partículas esféricas con el tiempo, a una profundidad determinada, cuando las mismas están sedimentando.

\section{MATERIAL Y METODOS}

\section{Modelo teórico}

El análisis de la distribución de partículas del suelo se hace, generalmente, por sedimentación en agua. Esta técnica se basa, como ya mencionáramos, en la ley de Stokes, siendo los dos métodos más difundidos el del hidrómetro de Bouyoucos y el de la pipeta de Robinson (Gee \& Bauder, 1986). En ambos casos lo que se busca medir es la concentración de partículas dispersas del suelo por unidad de volumen de la suspensión. Si suponemos que los tamaños de estas partículas esféricas están distribuidos según el modelo de fragmentación probabilística de Turcotte (1986), podemos combinar las ecuaciones 6 y 9 de la siguiente forma:

$$
\frac{\mathrm{M}(\mathrm{r}<\mathrm{R})}{\mathrm{M}_{\mathrm{T}}}=\left[\frac{1}{\mathrm{R}_{\mathrm{Lsup}}^{2}}\left(\frac{9 \eta \mathrm{h}}{2 \mathrm{~g}\left(\rho_{\mathrm{c}}-\rho_{\mathrm{L}}\right) \mathrm{t}}\right)\right]^{\frac{3-\mathrm{D}}{2}}=\mathrm{a}\left[\frac{1}{\mathrm{t}}\right]^{\frac{3-\mathrm{D}}{2}}
$$

donde $\mathrm{R}_{\mathrm{Lsup}}$ es el límite superior de radio de la esfera equivalente, $\eta$ es la viscosidad absoluta del líquido de la suspensión, h es la distancia recorrida por las esferas en el tiempo $t$, g es la aceleración gravitatoria y $\rho_{\mathrm{c}} \mathrm{y} \rho_{\mathrm{L}}$ son las densidades de la esfera y del líquido, respectivamente. Fijado h, a es una constante.

La Ec. 10 es una función potencial y por ajuste con los datos experimentales obtenidos mediante la técnica de Bouyoucos o de la pipeta de Robinson se podría verificar si la distribución de partículas en sedimentación tiene un comportamiento fractal y obtener también la dimensión fractal de fragmentación.

\section{Verificación experimental}

Para la verificación del correcto funcionamiento del modelo se usaron dos tipos de datos experimentales: a) datos propios y b) datos tomados de la literatura.

Para obtener los datos propios, las muestras de suelos estudiadas se extrajeron de un Argiudol Típico de la Estación Experimental J. J. Hirschhorn dependiente de la Facultad de Ciencias Agrarias y Forestales de la UNLP, situada en La Plata, provincia de Buenos Aires (Latitud: $34^{\circ} 55^{\prime}$, longitud: $57^{\circ} 50^{\prime}$ ), Argentina. La textura del horizonte superficial (Ap) es franco limosa. Se tomaron muestras con cilindros de $100 \mathrm{~mm}$ de diámetro y $200 \mathrm{~mm}$ de altura. Los sitios muestreados fueron cuatro, unos $100 \mathrm{~m}$ alejados entre sí. En cada sitio se tomaron dos muestras, las cuáles se llevaron al laboratorio, se dividieron a las profundidades de 0-100 $\mathrm{mm}$ y 100-200 $\mathrm{mm}$ y se dejaron secar a la temperatura ambiente. Posteriomente, se fragmentaron y mezclaron las dos muestras de cada sitio, para la misma profundidad, y se pasaron por un tamiz de $2 \mathrm{~mm}$, para realizar el análisis textural. Este se realizó utilizando el método de sedimentación en agua, con el hidrómetro calibrado según Bouyoucos (1962). De las mezclas de muestras de suelos de las mismas características se utilizaron $50 \mathrm{~g}$ para las medidas. El tratamiento previo de las muestras, para la dispersión de las partículas, fue el convencional (Gee \& Bauder, 1986). Los tiempos utilizados para medir la densidad de la suspensión acuosa fueron $40 \mathrm{~s}$, y 2, 8, $15,30,60,120,240$ y $1440 \mathrm{~min}$. La determinación de las densidades según Bouyoucos fue hecha por duplicado, y se tomó el promedio de las medidas para la determinación de la dimensión fractal mediante el ajuste de una curva 
potencial que relacionó el diámetro de las partículas y el porcentaje acumulado que permanecía en la suspensión acuosa.

Los datos de la literatura fueron tomados de Kaddah (1974). Este autor estudió muestras superficiales de veinticuatro suelos con diferentes texturas, mediante los métodos de la pipeta de Robinson y del hidrómetro de Bouyoucos. De los resultados publicados por este autor se eligieron los 24 suelos cuya textura fuera medida por el primer método. La dependencia del porcentaje de partículas variando con el tiempo fue obtenida por interpolación usando la Fig. 1 de la publicación, de acuerdo a lo sugerido por el autor.

\section{RESULTADOS Y DISCUSIÓN}

En la Figura 1, se puede observar una representación típica de la fracción de masa de partículas acumulada en suspensión, obtenida en este estudio, versus el tiempo de sedimentación. Estos datos experimentales fueron ajustados mediante regresión no lineal con la Ec. 10 desarrollada en este trabajo. En la Figura 2 se muestra el mismo conjunto de datos experimentales, pero la fracción de masa se ha representado en función del diámetro equivalente de las partículas. También en este caso se hizo un ajuste de los datos con una función potencial, la Ec. 6, obtenida de Tyler \& Wheatcraft (1992). Las dimensión fractal obtenida de ambas ecuaciones es conceptualmente la misma. Los valores de D obtenidos mediante la Ec. 6 dio, en efecto, resultados similares (no reportados en este trabajo), dentro de un 1,5\% respecto a los obtenidos mediante la Ec. 10. En la Tabla 1 se pueden ver los valores de D obtenidos en este trabajo a partir de nueve datos medidos, para los suelos de Argentina, utilizando la Ec. 10, con los correspondientes coeficientes de determinación, $\mathrm{R}^{2}$. La variación de estos coeficientes, entre 0,927 y 0,992 , da una idea del buen ajuste logrado por el modelo utilizado.

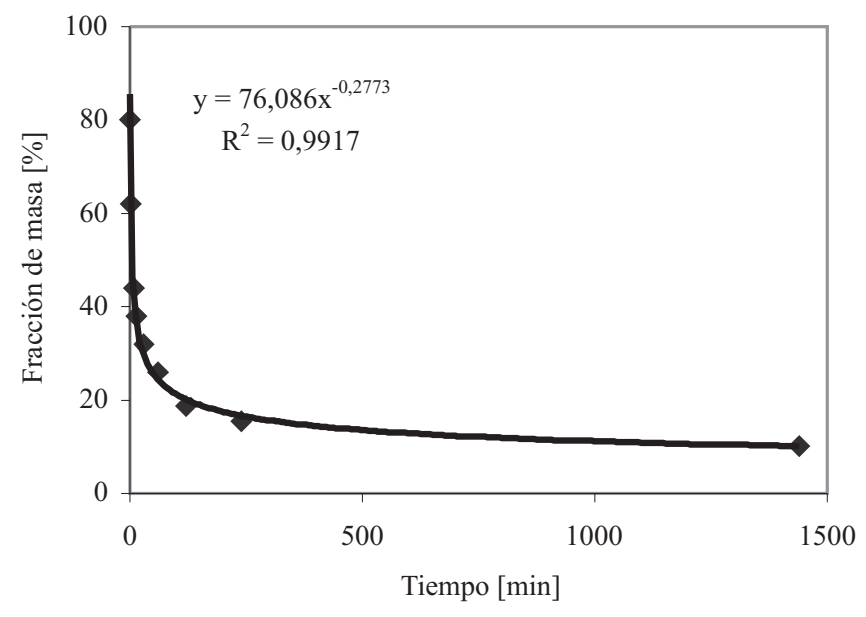

Figura 1. Representación gráfica de la fracción de masa de partículas en suspensión versus el tiempo (Ec. 10). El resultado del ajuste por regresión no lineal (línea continua) se hizo con los datos experimentales ( $)$ del sitio 4 de La Plata, Argentina

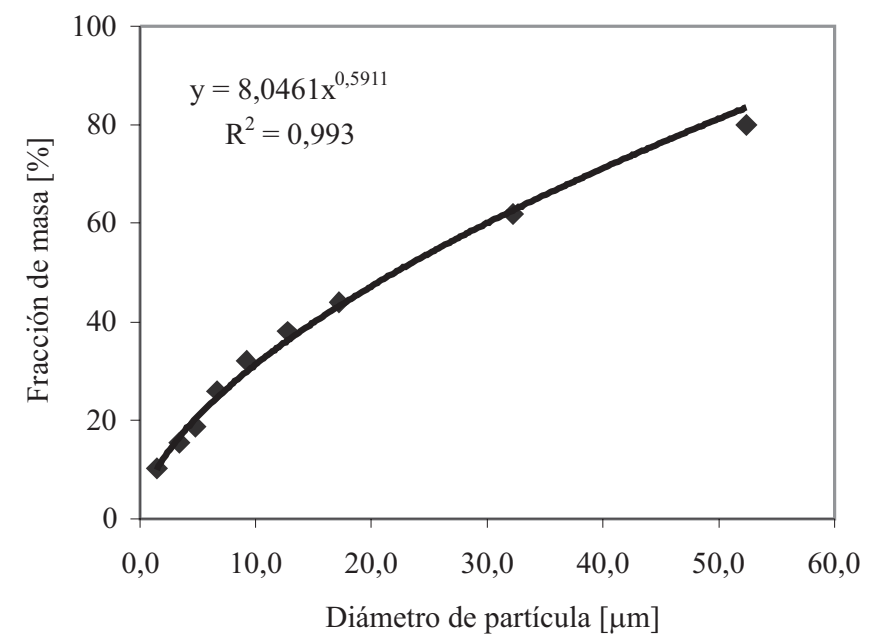

Figura 2. Representación gráfica de la fracción de masa de partículas en suspensión versus el diámetro de partícula (Ec. 6). El resultado del ajuste por regresión no lineal (línea continua) se hizo con los datos experimentales ) del sitio 4 de La Plata,

Tabla 1. Dimensiones fractales de fragmentación, obtenidas mediante el ajuste por regresión no lineal entre el porcentaje de partículas de un suelo remanentes en suspensión, en función del tiempo de sedimentación. También se pueden observar los coeficientes de ajuste $\mathrm{R}^{2}$. El suelo estudiado es de La Plata, Argentina

\begin{tabular}{ccccccccc}
\hline \multirow{2}{*}{ Prof [mm] } & \multicolumn{2}{c}{ Sitio } & \multicolumn{2}{c}{ Sitio 2} & \multicolumn{2}{c}{ Sitio 3 } & \multicolumn{2}{c}{ Sitio 4 } \\
\cline { 2 - 9 } & $\mathbf{D}$ & $\mathbf{R}^{2}$ & $\mathbf{D}$ & $\mathbf{R}^{2}$ & $\mathbf{D}$ & $\mathbf{R}^{2}$ & $\mathbf{D}$ & $\mathbf{R}^{2}$ \\
$0-100$ & 2,404 & 0,961 & 2,468 & 0,984 & 2,422 & 0,980 & 2,445 & 0,992 \\
$100-200$ & 2,431 & 0,966 & 2,481 & 0,927 & 2,483 & 0,987 & 2,512 & 0,988 \\
\hline
\end{tabular}

En las Figuras 3 y 4 se pueden ver representaciones gráficas de la fracción de masa versus tiempo y versus diámetro, para uno de los suelos de California (Kaddah, 1974). En este caso se usó la fracción limo, rango de 20 a $2 \mu \mathrm{m}$, con el fin de tener partículas pertenecientes a un sólo dominio de diámetros. En la Tabla 2 se pueden observar los valores de D estimados con la Ec. 10, y los coeficientes de determinación $\mathrm{R}^{2}$. El ajuste logrado en este

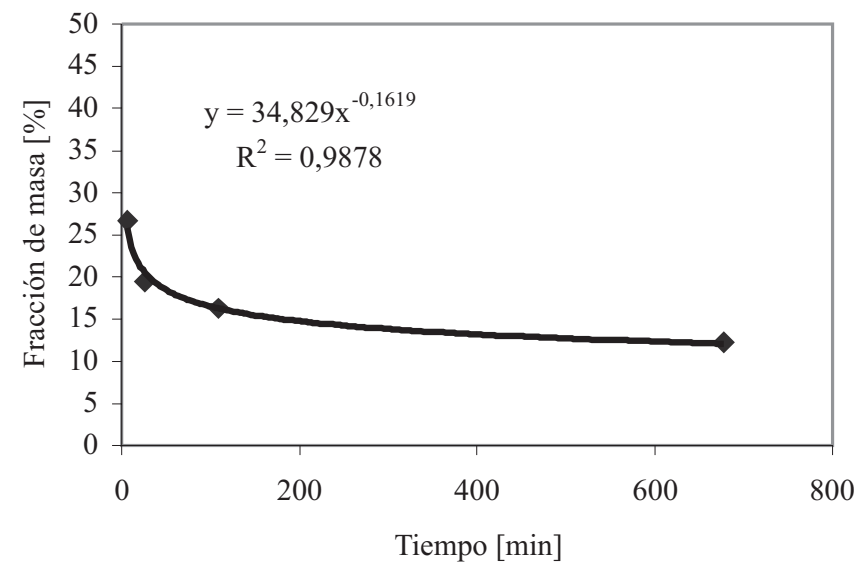

Figura 3. Representación gráfica de la fracción de masa de partículas en suspensión versus el tiempo (Ec. 10). El resultado del ajuste por regresión no lineal (línea continua) se hizo con los datos experimentales ( $)$ del suelo 14 de California, Estados Unidos de Norteamérica (Kaddah, 1974) 


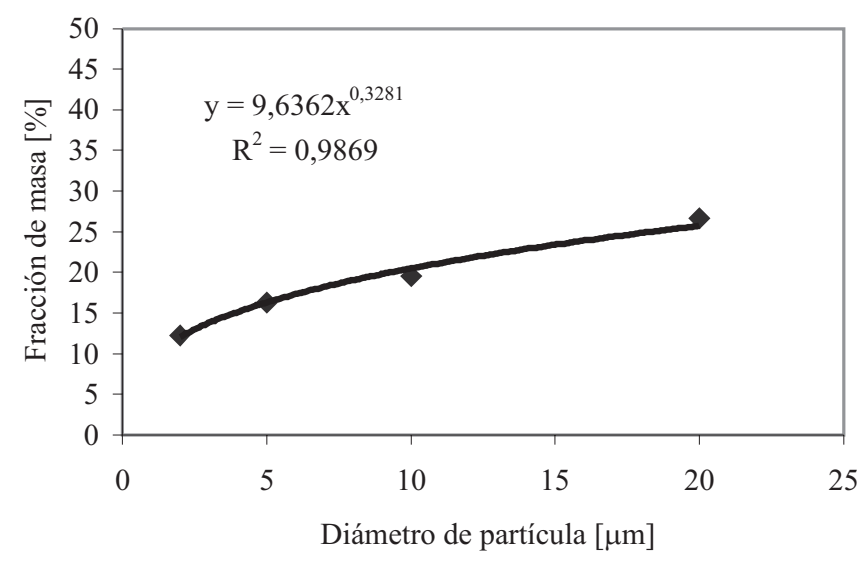

Figura 4. Representación gráfica de la fracción de masa de partículas en suspensión versus el diámetro de partícula (Ec. 6). El resultado del ajuste por regresión no lineal (línea continua) se hizo con los datos experimentales ( ) del suelo 14 de California, Estados Unidos de Norteamérica (Kaddah, 1974).

caso con cuatro datos experimentales, fue también muy bueno. También en este caso los resultados del ajuste de los datos experimentales mediante la Ec. 6 se encuentran dentro un $1,5 \%$ de diferencia respecto a los obtenidos mediante la Ec. 10. Los valores de los coeficientes de ajuste, $\mathrm{R}^{2}$, variaron entre 0,900 y 0,996 .

Se observa, en el caso del suelo de Argentina, que la dimensión fractal en la superficie, de 0 a $200 \mathrm{~mm}$, toma

Tabla 2. Dimensiones fractales de fragmentación, obtenidas mediante el ajuste por regresión no lineal entre el porcentaje de partículas de suelos remanentes en suspensión, en función del tiempo de sedimentación. También se pueden observar los coeficientes de ajuste $\mathrm{R}^{2}$. Las suelos estudiados corresponden a California (Kaddah, 1974)

\begin{tabular}{lccc}
\hline \multicolumn{1}{c}{ Suelo } & D & $\mathbf{R}^{2}$ \\
franco arcillo limoso & 1 & 2,686 & 0,996 \\
franco arcillo limoso & 2 & 2,666 & 0,989 \\
franco limoso & 3 & 2,523 & 0,943 \\
arcillo limoso & 4 & 2,696 & 0,995 \\
franco arcillo limoso & 5 & 2,560 & 0,989 \\
franco arenoso & 6 & 2,747 & 0,973 \\
arcillo limoso & 7 & 2,615 & 0,993 \\
franco arcillo limoso & 8 & 2,718 & 0,992 \\
franco arcillo limoso & 9 & 2,711 & 0,994 \\
franco limoso & 10 & 2,608 & 0,957 \\
Franco & 11 & 2,676 & 0,964 \\
franco limoso & 12 & 2,466 & 0,975 \\
franco limoso & 13 & 2,500 & 0,988 \\
franco & 14 & 2,676 & 0,988 \\
arcillo limoso & 15 & 2,775 & 0,990 \\
arcilla & 16 & 2,806 & 0,961 \\
franco limoso & 17 & 2,434 & 0,944 \\
franco limoso & 18 & 2,578 & 0,944 \\
franco limoso & 19 & 2,670 & 0,983 \\
franco limoso & 20 & 2,586 & 0,907 \\
franco limoso & 21 & 2,693 & 0,920 \\
franco arenoso & 22 & 2,819 & 0,995 \\
arenoso franco & 2,791 & 0,951 \\
franco limoso & & & 0,900 \\
\hline
\end{tabular}

valores entre 2,404 y 2,512. Para los suelos estudiados por Kaddah (1974) el rango de dimensiones fractales obtenidas varió entre 2,434 y 2,819. Tanto Wu et al. (1993) como Borkovec et al. (1993) sugirieron un valor universal $\mathrm{D}=2,8 \pm 0,1$, lo cual está fuera del rango obtenido en este trabajo. Bitelli et al. (1999) reportaron, para lo que ellos denominaron el dominio limo, valores de D en el rango $1,728-2,792$, siendo los valores más altos correspondientes a suelos con mayor contenido de arcilla, es decir suelos que presentan una distribución de partículas más finamente particionada. Filgueira et al. (2002) trabajando en el dominio limo, encontraron que la dimensión fractal de fragmentación variaba entre 2,410 y 2,679, siendo los valores correspondientes a muestras de mayor profundidad.

\section{CONCLUSIONES}

1. El modelo de sedimentación propuesto para un conjunto de partículas que tienen una distribución fractal de masa en función del diámetro, que obedecen la ley de Stokes, da también como resultado una función potencial de la fracción acumulada de partículas que permanecen en la suspensión versus el tiempo de sedimentación.

2. La ecuación resultante ajusta adecuadamente los datos experimentales obtenidos por el método de Bouyoucos o de la pipeta de Robinson.

3. La dimensión fractal obtenida mediante la nueva ecuación es la misma que resulta del modelo de fragmentación de Turcotte (1986). Los valores que toma esta dimensión es variable y dependiente del tipo de suelo.

\section{LITERATURA CITADA}

Baveye, P.; Boast, C. W. Concepts of "fractal" in soil science: demixing apples and oranges. Soil Science Society of America Journal, Madison, v.62, n.5, p.1469-1470, 1998.

Bitelli, M.; Campbell, G. S.; Flury, M.. Characterization of particle-size distribution in soil with a fragmentation model. Soil Science. Society of America Journal, Madison, v.63, n.4, p.782788, 1999.

Borkovec, M.; Wu, Q.; Degovics, P.; Laggner, P.; Sticher, H.. Surface area and size distributions of soil particles. Colloids and surfaces A: Physicochemical and Engineering Aspects, Amsterdam, v.73, n.1, p.65-76, 1993.

Bouyoucos G. J. Hydrometer method improved for making particle size analysis of soils. Agronomy Journal, Madison, v.54, n.3, p.464-465, 1962.

Filgueira, R. R.; García, M. G.; Roggiero, M. F.; Cerisola, C.; Aragón, A.; Sarli, G. O; Soracco G. Caracterización de la distribución de tamaño de partículas en suelos con un modelo fractal. En: Congreso Argentino de la Ciencia del Suelo, 18, 2002, Resúmenes... Puerto Madryn: AACS, 2002a, v.1, p.13

R. Bras. Eng. Agríc. Ambiental, v.10, n.2, p.277-282, 2006. 
Filgueira, R. R.; García, M. G.; Roggiero, M. F.; Cerisola, C. I.; Aragón, A.; Sarli, G. O. Uso del modelo fractal para caracterizar la distribución de tamaño de partículas en suelos. Ciencia del Suelo, Santa Rosa, v.20, n.2, p.114-117, 2002b.

Gee, G. W.; Bauder J.W. Particle-size analysis, in A. Klute (ed.) Methods of soil analysis. Part 1. Physical and mineralogical methods, 2.ed., Madison: ASA-SSSA, 1986, p.383411. Agronomy Monograph No.9

Kaddah, M. T. The hydrometer method for detailed particlesize analysis: 1. Graphical interpretation of hydrometer readings and test of method. Soil Science, Baltimore, v.118, n.2, p.102-108, 1974.
Mandelbrot, B. B. The fractal geometry of nature. New York: Freeman \& Co.:1982. 468p.

Stokes, G. G. On the effect of the lateral friction of fluids on the motion of pendulums. Transactions of Cambridge Philosophical Society, Cambridge, v.9, n.1, p.8-106, 1851.

Turcotte, D. L. Fractals and fragmentation. Journal of Geophysical Research, Washington, v.91, n.2, p.1921-1926, 1986.

Tyler, S. W.; Whitecraft, S. W. Fractal scaling of soil particle size distributions: analysis and limitations. Soil Science Society of America Journal, Madison, v.56, n.2, p.362-369, 1992.

Wu, Q.; Borkovec, M.; Sticher, H. On particle-size distributions in soils. Soil Science Society of America Journal, Madison, v.57, n.4, p.883-890, 1993. 